\title{
Authority and Hierarchy within Anonymous Internet Relay Chat Networks
}

\section{by Stewart K. Bertram}

\section{(cc) $\mathrm{BY}$}

This work is licensed under a Creative Commons Attribution 3.0 License.

\begin{abstract}
The protest group Anonymous has emerged as one of the most significant social movements of recent years, announcing their arrival as a globally recognized brand with regular disruptive cyber attacks and the leak of large volumes of sensitive data. Despite the obvious significance of Anonymous, to date, little academic research has been focused on what role hierarchy and internal authority play (if any) within the group. Bespoke software was used to structure over 5000 examples of IRC log files, so that a base line descriptive statistical picture could be drawn, as well as a more granular enumeration of the privileges associated with individual users.
\end{abstract}

Keywords: Anonymous, Social Media Monitoring, Internet Relay Chat, Online Community, Leaderless resistance movement, Online protest group

\section{Introduction}

The close of 2012 saw Anonymous named by Time magazine as one of the top 100 most influential people active within the world today (Time, 2012). The Time magazine article is the culmination of the events of the past three years that has seen a string of high profile hacking exploits by the Anonymous Collective. Incidents such as the mass Distributed Denial of Service (DDOS) attack on PayPal in response to the suspension of financial services to Wikileaks in 2010 and the numerous incidents of the unauthorized release of gigabytes of sensitive data, has changed the perception of Anonymous, from puerile pranksters to serious political actors (Chomsky interviewed by McGrain circa 2012)

As the Anonymous Collective have developed from a minor special interest group into a global protest group so their use of technology has kept pace with their social evolution. From localised pockets of Anonymous members, confined to obscure Internet bulletin boards like 4 Chan, evidence of Anonymous activity is now obvious on more mainstream sources of social media like Facebook, Twitter and MySpace. Conjoined within this increasing record of Anonymous activity is the assertion that Anonymous is leaving behind its minority routes and fast becoming a fully-fledged social movement (Halupka, 2011).

Despite the fact that Anonymous has always disavowed the existence of any kind of leadership structure within the Collective, there has often been an innate assumption within those examining the group that both explicit, and more suitable forms of hierarchy exist within Anonymous. Certainly, studies such as Olsons We Are Anonymous (2012) showed a body of evidence that Anonymous has a strong (if unconventional) hierarchy active within it.

The primary focus of Olsons work was the Internet Relay Chat(IRC) [This and other common terms used within hacking circles are defined in Annex B] servers that provide a peer-to-peer, instant messaging service that acts as a hub for many Anonymous members to socialize and coordinate their actions. The Anonymous IRC has been used on numerous occasions to launch and coordinate DDOS attacks on multiple targets 


\section{Journal of Terrorism Research}

and investigation by law enforcement agencies within the IRC and has led to a number of convictions of Anonymous members (Leyden, 2012). With the use of emotive language such as "ring leaders" (Parish, 2011) and "driving force" (Cockerton, 2011) being used by the Western legal system to describe convicted Anonymous members apprehended for their actions on IRC, the IRC is an obvious place to look for the possible existence of a hierarchy within Anonymous.

The main object of study of this paper is the Anonymous IRC, with the objective of gaining an insight into if there is a hierarchy active within Anonymous, and if there is how users exercise power over other users and if the majority of IRC users observes hierarchy.

\section{Literature}

This is not the first work to focus on either the social networks of computer hackers (Holt, et al, 2012) or Anonymous IRC as the main article of study. Olson (2012) examined in great detail the history, social dynamics and the wider lives of the actors involved in the IRC subculture of Anonymous. Olson's work although complete, is exclusively qualitative in nature, and although there are examples of quantitative studies being applied to socially deviant groups use of social media (Décary-Hétu et al, 2011), there is a well-defined gap within the literature for a study that examines Anonymous IRC in a quantitative way. Gabriel Colman's work into hacker communities, also stands as a notable corner stone for this project, however, much like Olson's work, Colman's work is exclusively qualitative in nature and does not explicitly examine the presence of authority within Anonymous IRC.

Debate is fast developing surrounding the presence of hierarchy within Anonymous. Early and with hindsight, flawed analysis by some cyber analysts (Barr referenced in Olson, 2012 page 4) concluded that Anonymous was a classic hierarchy, within which a small group of leaders leveraged the power of a much larger group of supports. The assumptions made concerning Anonymous's organisational form and the presence of a tangible leadership element with the group has most sharply manifested itself in the direction US and UK law enforcement has taken in prosecuting Anonymous members.

Despite the actions of the policing practitioner base, consensus opinion on the existence of hierarchy within Anonymous has by no means reached consensus, with events such as the Arab Spring (Ghonim, 2013) and the Occupy Movement (Chomsky, 2012) challenging the assumption that mass cyber protest movements necessitate overt hierarchical leadership. As Rid observes

"...the more a subversive movement relies on technologies and networked communication, the more difficult it will be to establish an internal coercive order." adding that "it is this internal order that differentiates a movement from a group." (Rid, 2013 page 131)

What distinguishes Anonymous from other protest groups is its almost zealous rejection of authority. Shown below is a typical statement common to many pieces of propaganda associated with Anonymous

"You cannot join Anonymous. Nobody can join Anonymous. Anonymous is not an organization. It is not a club, a party or even a movement. There is no charter, no manifest, no membership fees. Anonymous has no leaders, no gurus, no ideologists. In fact, it does not even have a fixed ideology (sic)." (Harriswow, 2012)

Anonymous is certainly not alone within the list of social movements who state a rejection for the need of hierarchy (Pearce 1980, Brown 1989, Lichterman 1995: 196). However, studies into social movements 


\section{Journal of Terrorism Research}

have long identified the need for some kind of internal organization to maintain cohesive collective action (Melucci, 1996: 344-7).

Possibly a better model for interpreting the social structure of Anonymous than the conventional concepts of social organization is to look at the commitment of Anonymous members to the activities of the Collective (commitment in this case being defined as the time invested in group activity). Broadly speaking the most prominent Anonymous members are the most committed members, spending hours of time in IRC chat rooms and generally getting deeply involved in the culture of Anonymous (Stryker, 2011 page 60).

\section{Method}

Technology is the lifeblood of computer hackers, as Castelles comments

“...serious hackers, primarily exists as hackers on-line" (Castelles, 2001).

As such the primary focus of the research revolved around adding structure to 5355 IRC logs collected from the Anonymous IRC server within the data range of January to October 2012. Both quantitative and qualitative methods were used to analyze the data. Quantitative methods provided the base descriptive statistics that allowed a comprehensive visualization of the IRC landscape. Two sets of data were analyzed during the quantitative phase of research to derive statistical measures. Set One comprised the 5355 logs of the IRC, from which baseline descriptive statistics were drawn, while a smaller subset of 1104 logs (three consecutive months worth of data) were used to derive statistics on hierarchy. The subset of 1104 logs was used due to the greater volume of individual measures that the software produced when examining hierarchy, with the rational that a smaller set of variables would allow dependencies and relationships between variables to be seen more clearly. Qualitative methods were used to add analytical depth to the statistics.

The justification for confining the scope of the study to the Anonymous IRC, was that taken collectively, the Anonymous social media landscape sprawls to the extent that gathering each of the possible sources together, in a representative way, is beyond the capabilities of all but the most resourced research projects. Instead this study focuses on Anonymous IRC as it acts as a bounded medium, which is anecdotally credited with being the centralised command and control loci for the Anonymous Collective (Khumar, 2012). As such the assumption of the research is that by focusing on the IRC as one key aspect of Anonymous, a meaningful analytical result regarding the groups social dynamics could be derived.

A cursory examination of the IRC shows evidence of the possible presence of hierarchy in the form of privileges, which are assigned by channel administrators to channels users. A privilege manifests itself in a graphical form with a symbol displayed in front of the nick (shown below)

[01:47:23] @uzzy> Sorry no one spoke in the minute you joined.

Figure 1: Example of privilege in IRC chat (the highlighted @)

Initial test analysis of the data showed that there were six levels of privilege observable within the IRC (including non privilege), they are shown below 
The Centre for the Study of Terrorism and Political Violence

\section{Journal of Terrorism Research}

\begin{tabular}{|c|c|c|}
\hline Privilege & Example & $\begin{array}{c}\text { Associated } \\
\text { Graphic }\end{array}$ \\
\hline$\sim$ & $\langle\sim \mathrm{U}\rangle$ & $\mathbb{\Omega}$ \\
\hline$\&$ & <\&plumber3> & $\theta$ \\
\hline (a) & $<@$ Fish_man $>$ & A \\
\hline$\%$ & $<\%$ nix $>$ & \\
\hline+ & $<+E g g>$ & 团 \\
\hline No Privilege & $<$ BumedFlag $\rangle$ & NA \\
\hline
\end{tabular}

Figure 2: Privileges within the IRC forums

Additionally it was obvious that not all privileges are equal and an automatic hierarchy is hard coded into the technological architecture of IRC. Shown in Figure 3 is an example of privilege ordering within IRC. 


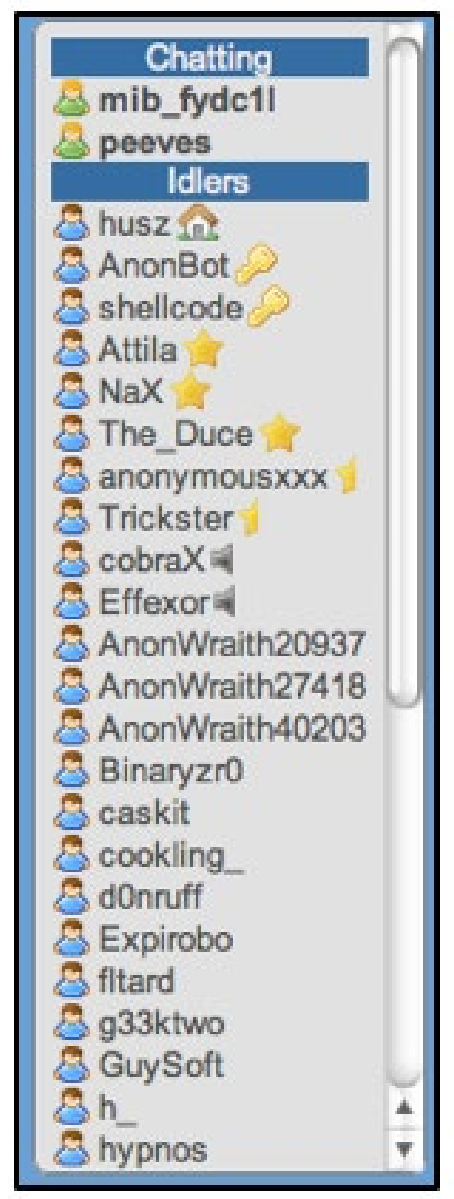

Figure 3: List of users active within an IRC channel and ordered according to privilege (represented by icons next to name). Most senior at the top, user with no privileges listed below

The image shown in Figure 3 is the display that a user sees when accessing the IRC and is a fixed feature of the technology.

Based upon examples such as the one shown in Figure 3, a working theory was developed that the privilege system was a substitute for authority within the IRC and it was this privilege structure that formed a hierarchy within the IRC. Based upon this assumption, software was developed that would structure the IRC logs so that key aspects of the data set could be enumerated, such as the number of privileges active within certain channels.

\section{Results}

Aside from the statistics concerning privilege some base line statistics concerning the data set are shown below (all statistical measures based on the larger set of 5355 logs)

- $\quad$ The mean average number of nicks per IRC log was 242

- $\quad$ The mean average number of posts per IRC log was 1592

- The greatest number of nicks recorded in one channel, in one 24hr period was 6043 - megaupload. Anops.20120119 (All dates YYYY/MM/DD format) 


\section{Journal of Terrorism Research}

- The greatest number of posts recorded in one channel, in one $24 \mathrm{hr}$ period was 31938 - Vox. VoxAnon.20121014

- $\quad$ The greatest post per hour rate in one channel, in one $24 \mathrm{hr}$ period was 3319 - Megaupload. Anops.20120119

- The greatest post per user in one channel, in one $24 \mathrm{hr}$ period was by AnonmyousWiki, in the channel opnewson.Vox.20120526, with over 26828 posts (all statistical measures taken from Bertram, 2013)

Aside from the basic descriptive stats shown above the principle objectives of the research was to understand how the privilege structure outlined earlier affected the social dynamic within the IRC. The first step in this process was to innumerate the presence of privilege within the data set. The two essential questions that this strand of the research sought to answer were

1. How is the hierarchical structure of the privilege system deployed across the IRC? i.e. what is the ratio of IRC channels that have fully developed privilege systems compared to those that have none?

2. How widespread are privileges across the Anonymous IRC? i.e. what are the ratios between nicks with privileges and those without?

Turning to the first question, shown below in Figure 4, is a break down of a sample of 1104 logs taken from the main data set. Each log has been scanned for the presence of nicks with privileges and scored accordingly. A score of 1 represents a log where no nicks with privileges are present, 6 represents a log were a full range of nicks with privileges are present ( , \&, @, \%, +, those with no privileges are also counted) the other scores represent various graduations of the presence of privileged individuals. The percentiles represent the number of logs showing a privilege grade in relation to the wider data set i.e. 11.6\% (127 logs) had three levels of privilege evident within them of the 1104 logs.

\section{Percentage brake down of privileges across $1104 \log$ files}

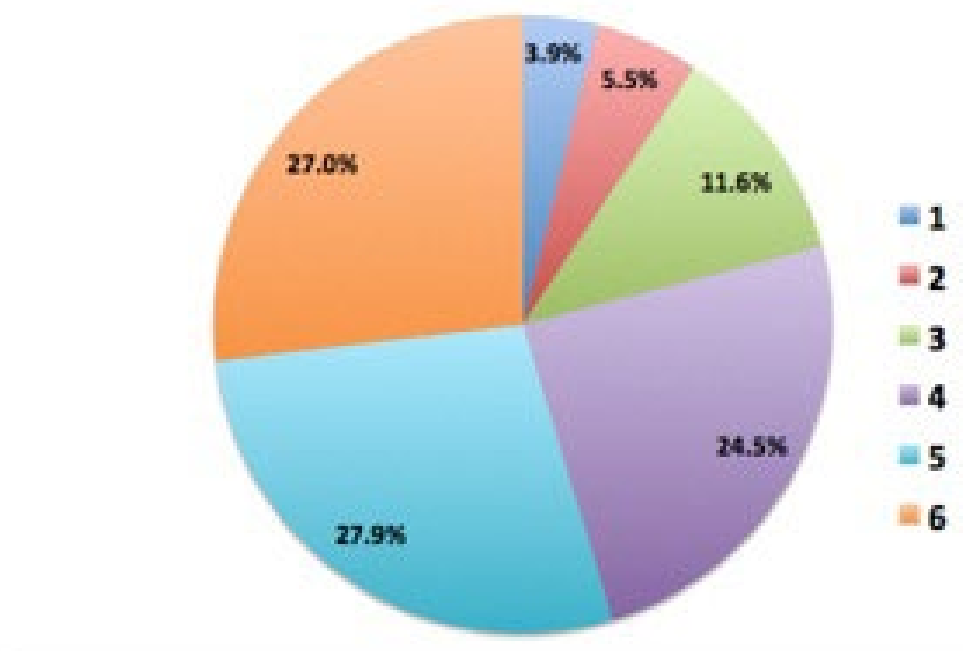

Figure 4: Privileges brake down per log file

The clear conclusion that can be drawn from Figure 4 is that over half the logs within the sample data set have well-developed system of privilege evident within them, with only $3.9 \%$ (42) logs showed a completely 


\section{Journal of Terrorism Research}

flat privilege structure. The logs that had no privilege structure were generated from channels for the most part that were transient within the IRC, had little posting activity or developed a privilege structure after a few days of inactivity.

Adding context to the results present in Figure 4 is the bar graph displayed in Figure 5, which shows the number of nicks active within the data set, segregated by the privilege score of the IRC log they were active within e.g. 298 logs had a score of 6 (full privilege structure) and 100692 nicks were associated with the 298 logs.

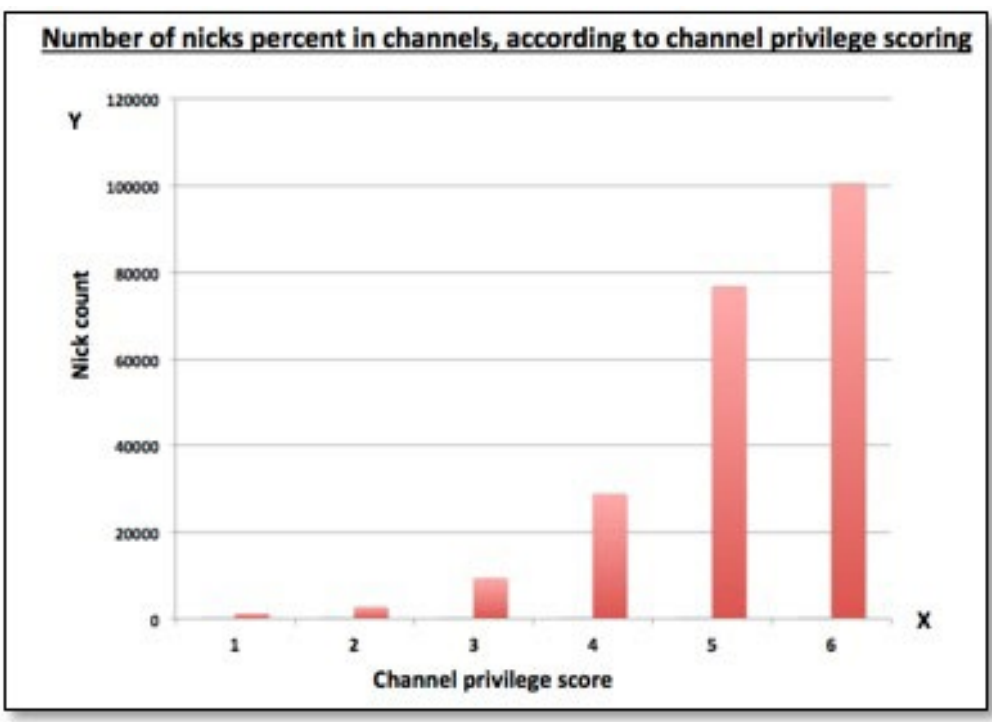

Figure 5: nick to privilege score

Taking the results of Figure 4 and 5 together there is a strong suggestion that where Anonymous members cluster they create a system of privilege and the more members there are active within a channel the more fully developed the privilege structure becomes.

An analytical step further than looking at the distribution of privilege over the IRC is to examine how privileges relate to each other within different channels. Shown below in Figure 6 is a graph showing average count of privileges within a selection of randomly selected channels taken from the data set (the nick count for the actors with no privileges was removed from this visualisation due to size issues). 


\section{Journal of Terrorism Research}

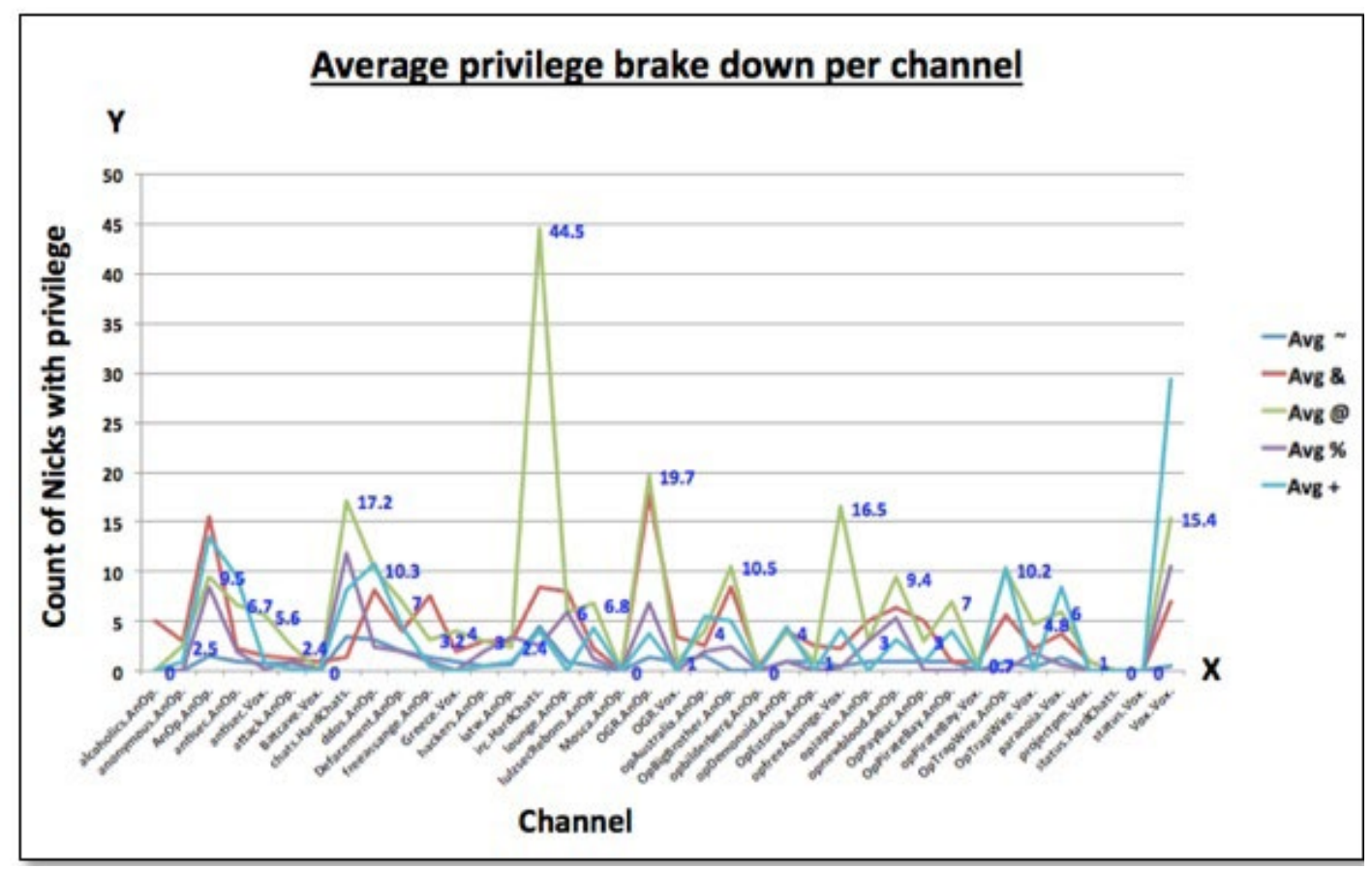

Figure 6: nick to channel count, broken down by assigned privilege

The chart shown above gives a number of insights into the relationship between privileges within Anonymous IRC.

Somewhat expectedly the highest rank in the privilege structure $(\sim)$ is typically the least common in most channels, with the other subordinate privileges $(\&, \%,+)$ roughly trending upwards in number in accordance to the $\sim$ count. This pyramid form of privilege distribution, mirrors the form of a conventional hierarchy based organisational structure (Itoh, 1992).

There are a number of surprising anomalies regarding privilege within the data set, two of which are apparent in Figure 7 (below) namely, the huge spike in the @ privilege within the IRC.Hardchats channel and the spike in the $\sim$ privilege in the Vox.Voxanon channel. This led the researcher to consider if some channels are acting as more centralised command and control hubs for the wider IRC or if certain channels were conducting specific activities that attracted a specific concentration of privileged individuals. Both of these concepts are explored in more detail in the next chapter.

Another highly surprising trend within the data regarding privilege was the relationship (or lack thereof) between the number of privileged nicks within a channel and the count of nicks with no privileges. Shown below are two visualisations of the nick count of the privileged (left) compared to the count of the nicks with no privileges (right). 


\section{Journal of Terrorism Research}

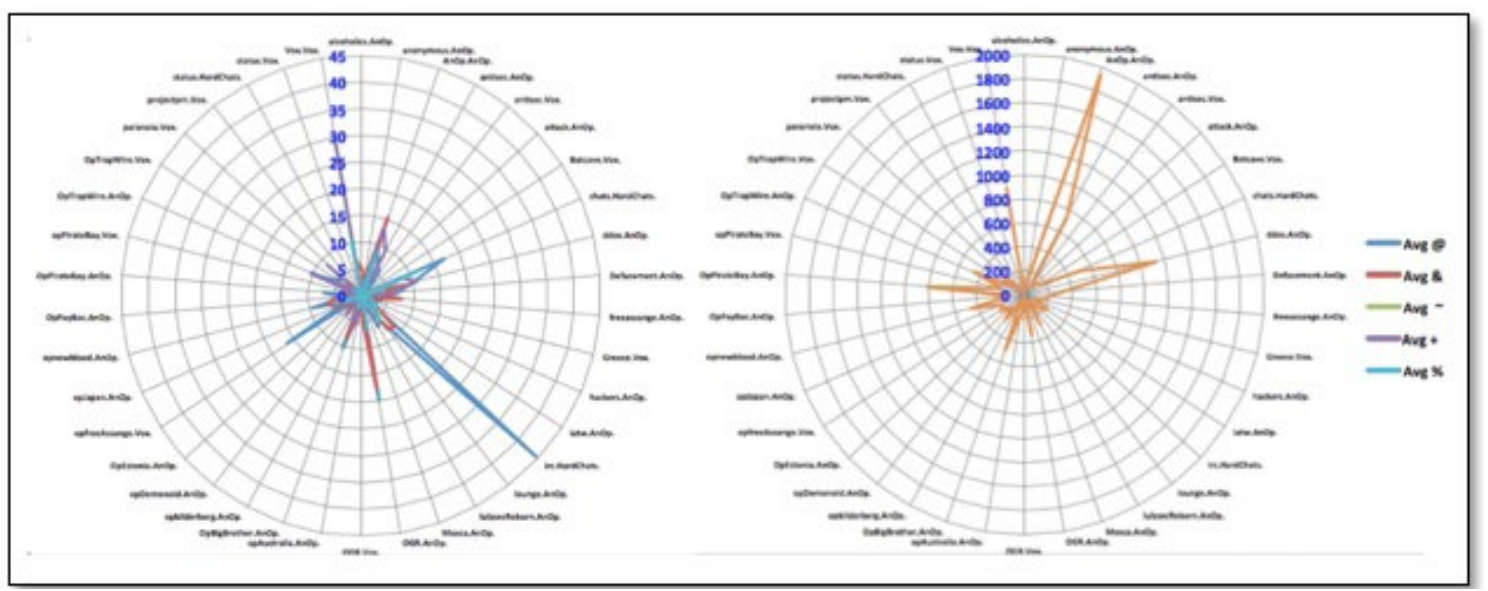

Figure 7: Two visualisations of the average privileged and non-privileged nicks active within the same channels (note the scale shown in blue on each graph)

Clear from Figure 7, is the huge disparity between privileged and non-privileged nicks within the same channel. A more formal R(squared) plot of the relation between privileged and non-privileged nicks, shows none or a loose correlation between overall number of nicks active within a channel and the number of privileged individuals active within that channel.

Based on the data shown in Figure 7, the obvious question arises: if one conceptualizes Anonymous IRC as a classic hierarchical structure of command and control, how much influence could such small numbers of privileged nicks have over the wider community?

\section{Transient nicks, static hierarchy}

With the above question in mind, the data was analysed with the intention of discovering how static nicks with privileges were, in relation to individual IRC channels, compared to the nicks with no privileges.

The data used for this section of the research was created from samples taken from four date ranges from three IRC channels. The data sampled focused on nicks and their attached privileges and if they posted to any of the three channels on any of the date ranges.

The analytical method applied to the data was Social Network Analysis, with the objective of quantifying how fixed privileged nicks were within the IRC landscape. A sample of the results generated from this effort are shown below in Figure 8 


\section{Journal of Terrorism Research}

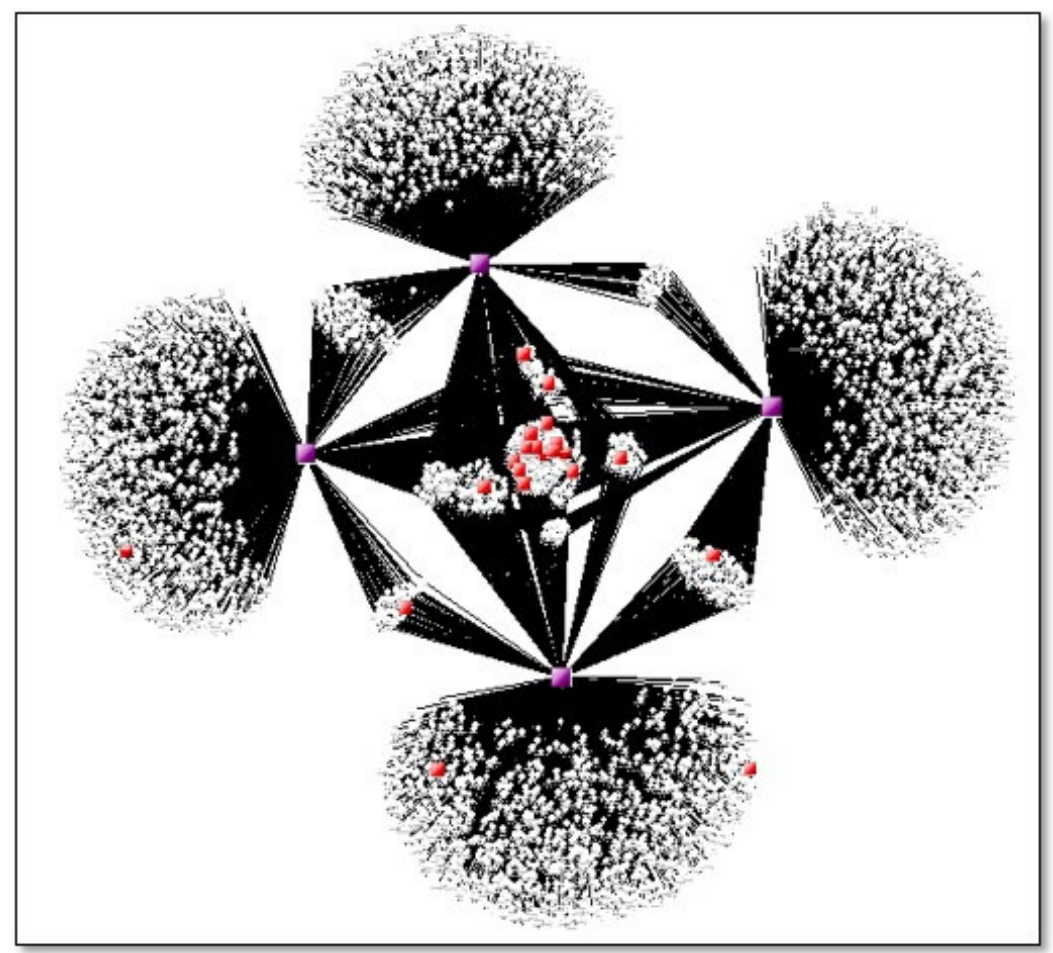

Figure 8: Social Network Graph, showing nicks active in the Vox. VoxAnon channel on four separate dates 1,7,14,21 October (dates represented by pink squares). White dots represent nicks with no privileges; red dots represent nicks with privileges.

The points that Figure 8 demonstrates is that while non-privileged nicks are highly transient within the channel (shown by the lack of interconnectedness between the four dates), nicks with privileges within the channels are far more static (demonstrated by their high degree of connectedness within the four dates).

The other two channels within the data sample set showed a similar pattern of fixed privileged nicks and transient non-privileged nicks.

Rid observed the "membership mobility," (Rid, 2013 page 150) of online groups adding that "the more a subversive movement relies on multiple causes, new technologies, and networked communication, the more likely it is that this movement will be characterised by high membership mobility" (Rid, 2013 page 127/128).

Looking at the results presented by the analysis thus far, the data would appear to confirm Rid's assertion regarding the transient nature of membership of an online group and suggest that the relationship between privileges and non-privileged nicks plays an important part in IRC life.

However, conceptualizing IRC privilege as a manifestation of traditional rank is misleading, instead the results of Figure 8 suggest that the privileged nicks in the Anonymous Collective act more as showmen attempting to attract support from an ever changing crowd, rather than commanders leading an army of followers. Additionally, it is incorrect to assume that all privileged members are ideologically aligned; instead at any one time there are a multitude of different privileged individuals advocating different (if not competing) causes within the IRC. 


\section{Journal of Terrorism Research}

\section{Qualitative Analysis Results}

Rid observed that "The more technologically sophisticated a subversive movement... the more cause driven it is likely to become" adding that "Rather than grand narratives, it is highly specific issues that are likely to mobilise a critical mass of enraged activists" (Rid, 2013 page 123)

The cause, or the operation as it is more commonly termed within Anonymous, is the cornerstone of many Anons engagement with the group. Anonymous operations such as Operation/ Project Chanology (anonbase, 2011), Op Avenge Assange and Op PayPal (Correll, 2010) have gained near legendary status within the cyber security world and the concept of an operation has become so central to Anonymous culture that the form of the IRC is structured around operations (only two of the channels recorded within the data set of this study (\#DDOS and \#Journalist) were not themed around an operational concept).

Statistics alone do not reflect the passion for the cause and sheer exuberance in their actions that many Anons feel towards their engagement with Anonymous, as such in contrast to the initial stages of the research this stage adopts an alternate view on the data by applying qualitative methods to portions of the data set.

Adopting a case study approach, the research profiles two specific IRC channels, Op Green Rights and Op Australia. These two operations were chosen as they stand at the poles of the Anonymous IRC culture with one being small scale and highly regional (Op Australia) and one being far more mainstream and globally based (Op Green Rights).

\section{Operation in Profile One - Op Green Rights}

Op Green Rights is an IRC channel and Anonymous operation that focuses exclusively on environmental issues. The channel is popular within the wider context of the IRC landscape and the data shows that the Op Green Rights IRC channel is always within the top five most visited Anonymous channels and frequently in the top three most visited channels within the IRC.

Op Green Rights averages 121 nicks visiting the channel per 24 hour period. Additionally, over $90 \%$ of the 52 Op Green Rights logs within the data set shows a fully or nearly a fully developed privilege structure. Surprisingly, given the previous statistical points, Op Green Rights has a low average posting number with just 129 posts per 24-hour period (other channels within the set of the top five most popular channels typically averaged between 800 and 1000 posts per $24 \mathrm{hrs}$ ).

The unusual posting activity within the Op Green Rights channel was reflected in the unusual nature of the discourse, with records from the channel showing particularly large numbers of nicks entering the IRC room and then almost immediately exiting and engaging in no dialogue during their period within the room. Dialog that did occur within the channel was characterised by intense bouts of highly technical conversation, generated exclusively from privileged nicks. An additionally observation concerning the interaction between nicks within Op Green Rights, was that dialogue occurred almost exclusively between privileged nicks with the non-privileged nicks within the channel being almost spectators to the wider channel dialog.

The topic of conversation would typically take the form of exultations to the other user within the channel to conduct DDOS attacks on a chosen target, with the target being nominated by the privileged nick. An example of this dialog is shown below 
[[09:22:55] < Aken>-check www.monsanto.com

[09:23:03] <\%Vots> It's not just you! http://www.monsanto.com looks down from here. [09:23:12] < Aken> come on ANON! little more

Figure 9: example of Op Green Rights IRC dialog

The example shown above is typical of the format of the wider dialog of IRC, with the initial observation that a web site is offline, followed by a conformation by another nick. As is typical with this type of dialog, the target web site (in this case www.monsanto.com) is not offline at all, merely under DDoS attack. Akens exaltation and encouragement of a "little more" is in reference to the need for more Anonymous members to participate in the DDoS attack to take the web site offline.

While many elements of the Op Green Rights dialogue were typical of the wider IRC landscape the dedication to a single target is unusual. Monsanto (a bio science company involved in the manufacture of Genetically Modified crops) was consistently targeted over a period of months by Op Green Rights, with many Monsanto web resources being successfully taken offline by Anonymous members working from the Op Green Rights channel.

Another aspect of the Op Green Rights channel that was immediately apparent was that almost all the nicks frequenting the channel were involved with a number of other Anonymous operations, and there seemed to be no identifiable group of nicks exclusively focusing on Op Green Rights as a cause. Even the most prolific posters such as Vots and Aken were more active in channels such as \#DDOS.

It was also apparent that the majority of the nicks within the channel was engaged in conversation on other technical platforms and IRC channels and used the Op Green Rights channel to continue ongoing conversations.

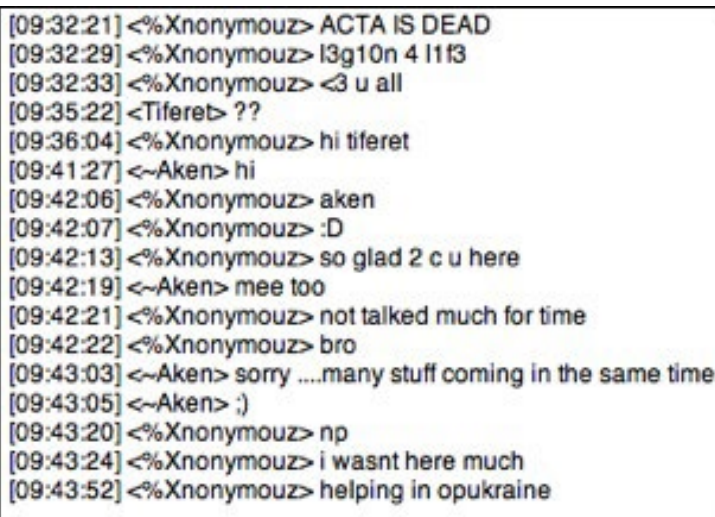

Figure 10: further example of Op Green Rights IRC dialog

Note in Figure 10, features such as the fact that dialogue is being engaged in almost exclusively by privileged nicks, the mention of a number of other operations (ACTA and OpUkraine) and the familiarity between nicks.

Op Green Rights is notable in that it is supported by an expansive blog that sits outside of the IRC landscape, and platforms such as this could act as meeting place for Anons interested in environmentalist issues. 


\section{Journal of Terrorism Research}

Another aspect of Op Green Rights that is unique to this channel, is the overt focus on data leeks in addition to DDoS attacks as an effective means of protest, note the example below

Figure 11: Op Green Rights IRC dialog talking about data leaks

The purpose of leaked data is to expose corporate malfeasance, and Op Green Rights shows a strong interest in not only stealing corporate data but analysing it and producing info graphics to more persuasively communicate the Op Green Rights message to the wider public.

Typical of almost all other Anonymous IRC channels was the persistent interest in social media, especially the micro blogging web site Twitter, with numerous examples of privileges members encouraging other Op Green Rights channels users to look at the Op Green Rights Twitter page. The curious situation of nicks encouraging other nicks to consume other types of social media, is an example of attempts by privileged Anons to network build in other areas of the social media space. Op Green Rights has a particularly strong presence in comparison to other Anonymous campaigns, on surface web platforms such as Facebook and Twitter and promoting these sites on IRC builds the support base when nicks "like" a page or web resource.

Qualitatively examining the Op Green Rights logs revealed a social structure that had little in common with any classic model of hierarchy. Instead privileged nicks seemed merely to be broadcasting suggestions to the wider IRC channel user group, with often little acknowledgment of their suggestions by other non-privileged Anons. Additionally the activities of the privileged would appear to be met with ambivalence rather than deference by the rest of the non-privileged IRC user group.

\section{Operation in Profile Two - Op Australia}

Op Australia, in contrast to Op Green Rights, is a regional based faction of Anonymous that is made up of individuals based in Australia and New Zealand. Again differing from Green Rights, Op Australia has no single issue that it pursues, instead the channel serves as a meeting space for Anonymous members, who due to the dint of the time zone they live in are isolated from the main body of the predominantly US and EU based Anonymous Collective.

Rather than being a single campaign, Op Australia periodically adopts issues that the group pursues or rejects based on levels of support, in effect; Op Australia is a microcosm of the wider Anonymous Collective.

An examination of the data generated from Op Australia IRC channel revealed almost immediately, the presence of a strong system of hierarchy and overt social control within the channel. After a more detailed examination of the data, it would appear that the decision-making within Op Australia fell to a leadership core of as little as five people.

- Juzzy - was identifiable as the overall leader of the Op Australia channel, displaying obvious behavior of rank such as delegating authority onto other member of the channel i.e. "Listen to Lorax and CiTRiX, [other Anons] they'll be in charge while I' am gone" and kicking other nicks that did not conform to the channels social norms (specifically one nick that took great pleasure in persistently 'slapping' Juzzy). Juzzys leadership position owed much to his technical hacking ability and willingness to demonstrate the best way to pursue Op Australia goals. Not a charismatic leader (Juzzy 


\section{Journal of Terrorism Research}

in fact rarely engaged in any form of extended dialogue), Juzzy effectively implemented the lead by example model of control within the channel.

- Lorax and CiTRiX - joint channel second in commands, with explicit authority delegated from Juzzy. This team were observably responsible for much of the day to day running of the Op Australia channel such as setting up social media outlets for the channel and conducting much of the cyber reconnaissance work that is required before the commencement of an attack. Lorax and CiTRiX also acted in a far more explicitly supportive way to other Anons than Juzzy, who openly directed technical questions away from himself and towards the pair.

- Husz - providing overt support in the form of consent to the leadership cabal of Juzzy, Lorax and CiTRiX, Husz was observed to follow the classic path of an aspirant Anonymous member. Initially developing a relationship with Juzzy, Husz in the end solidified his status within Op Australia by building a strong relationship with Lorax. Husz most often fulfilled to role of validating Juzzy's leadership though acts such as castigating the actions of nicks that Juzzy kicked.

While the author acknowledges that the organizational structures that were observed within the Op Australia logs, stopped short of the professional and bureaucratized organizational hierarchy that Zald (et al, 1973) identified as being key indicators of a mature social movement, there was a clear hierarchy active within Op Australia.

When examining the dynamics of authority within Op Australia's, a number of points became immediately apparent.

- The combined leadership group of Op Australia did hold sway over the flow of the discourse within the IRC channel. Collectively the leaders of Op Australia initiated and closed most conversations, enforced cultural norms and generally set the tone and pace of any cyber activity that Op Australia engaged in.

- Juzzy's leadership was not absolute, with regular challenges originating from other nicks to Juzzy's self-assumed status as operational commander. The two pillars that Juzzy's leadership was built upon were the continued support of his loyal lieutenants, Lorax and CiTRiX. The almost constant power struggle within Op Australia shows that any Anonymous operational commander can expect to have their authority challenged on a regular basis.

- In contrast to Juzzy's precarious situation as leader, Lorax and CiTRiX position within the Op Australia hierarchy seemed far more stable, with no apparent challenges to their social status being seen within the data.

\section{Conclusions that can be drawn from the Op Green Rights and Op Australia case studies}

- Many Anons maintain long term fixed personas within the IRC - this particularly manifest itself within the strong interpersonal relationships within Op Australia and the less intense, but platform spanning familiarity evident between the nicks in the Op Green Rights channel 


\section{Journal of Terrorism Research}

- Op Australia although seeming far more operationally coherent than Op Green Rights may have appeared so due to it being a much smaller scale operation involving fewer individuals. It would appear that the Op Green Rights IRC channel was just one aspect of a much larger movement

- It is obvious that authority is not automatically conjoined with privilege within Anonymous IRC. Adding to this statement are two sub observations concerning privilege and authority

- $\quad$ Privilege represents different things in different channels - within Op Australia privilege represents the manifestation of a classic system of hierarchy, while in Op Green Rights privilege would appear to merely represents the continuing presence of a nick within that channel

- Levels of Membership Mobility directly affects the levels of authority the privileged nicks can exert - within Op Australia, with its lower rate of Membership Mobility, the privileged nicks hold a greater sway over a more captive audience, while within Op Green Rights with its very high levels of Membership Mobility, privileged nicks are merely advocates for proposed actions rather than enforcers of cultural norms

Taking the above points together it is apparent that Membership Mobility and privilege are dependent variables when examining the role that the privileged play within an IRC channel.

Barr's research (summarized in Olson, 2012 page 5), conceptualized Anonymous as a purely hierarchical organization within which a handful $(<30)$ individuals made the decision that influenced the wider Collective. The research presented within this paper, challenges this conceptualization of Anonymous as overly simplistic.

The two case studies show that the Collective is neither completely a classic hierarchy nor a fully leaderless group; instead social organization within Anonymous is analogous to the relationship between the customers and the showmen within a circus.

The customers are analogous to the nicks, a constantly changing group of transient users, swirling around the circus attractions (the IRC channels). The showmen are the privileged within the IRC and their power is derived from their knowledge of Anonymous, quick wit and occasionally, technical computing skills. It is the privileged nicks oratory skills that allow them to attract other unprivileged nicks into becoming involved in an operation, in much the same way a showman attracts customers to a sideshow.

Analogising Anonymous IRC in this way redefines the role of the privileged, from conventional leaders, to factional actors engaged in a classic model of resource competition, with the main resource for Anonymous being the support of the un-privileged multitude.

Within this context, the role of the privileged becomes clearer and explains the disparity in privileged versus non-privileged counts observed within earlier in the research. It would appear that just as in a circus whichever showman / privileged nick presents the best show attracts the most attention.

As Diani comments

“...leadership roles need not entail control over a unified organization... They may also, far less obtrusively, result from certain actors' location at the center of exchanges of practical and symbolic resources among movement organizations. This will not generate domination...but rather varying degrees of influence" (Diani 2003: 106). 


\section{Journal of Terrorism Research}

From the two case studies of Op Green Rights and Op Australia it would appear that some factions of Anonymous follows Dianis' model of hierarchy, while some lean far more heavily towards a more classic authoritarian model of authority. One possible explanation for this is the varying degrees of membership mobility that each IRC channel observably has.

Linking with the results of the quantitative study outlined earlier, it is possible that high membership mobility could actively inhibit the creation of hierarchy within Anonymous. As authority appears to be derived organically in a large part from the persistence of an identity in a channel and the familiarity of that identity with other persistent users, high membership mobility could actively inhibit the functioning of this process.

Turning to the results of the data set, Op Green Rights has high membership mobility and a hierarchy however, there is no authority being openly demonstrated within the channel. Conversely Op Australia has low membership mobility and defined hierarchy within which authority is clearly demonstrated. While not enough to create a rigorous exploration of the idea the concept suggested by the data is that high membership mobility inhibits the creation of hierarchy within a hyper-networked group like Anonymous.

\section{Conclusion}

"...the group mind does not think in the strict sense of the word. In place of thoughts it has impulses, habits, and emotions. In making up its mind, its first impulse is usually to follow the example of a trusted leader." (Edward Bernay's 1928 page Propaganda 73)

This study of the Anonymous IRC has both confirmed and challenged Bernay's statement about the role the privilege plays within groups such as Anonymous. This study has found that privilege is endemic across the IRC landscape, with the majority of IRC channels showing a well-developed privilege structure. This study has also observed the classic presence of authority, symbolized by privilege, active within certain channels. As such it would appear, on first impressions, that this study unilaterally supports Bernay's statement and challenges Rids assertion that "internet-driven subversion... is characterised by lower levels of organisational control" (Rid, 2013 page 150). However, closer inspection of the data has shown as many examples of leaderless masses as led masses.

While groups with established leaderships would appear to be common within the IRC (shown by the ubiquity of privilege structure), groups with hierarchical structures would appear more to share a common space with non-privileged nicks, than provide an overall leadership function within an IRC channel.

As such one of the main findings of this study is that an Anonymous IRC channel is analogous to a common public space within which a multitude of groups gather. Some of the groups within the IRC channels have privilege structures but just as many groups do not. Continuing the public space analogy, not all groups within the IRC space have an inter group dialog. As such it is a mistake to assume that the social dynamic of an IRC channel is that of a leadership group providing direction to the wider user group, this is an oversimplification of a more complex situation. Instead, privileged groups are a result of a subset of users within the IRC that desire to be part of a hierarchical structure. While privilege does translate into authority in some cases (shown by the Op Australia profile) it would appear that most of the time, the authority of a privileged user does not extend outside of a relatively small circle of nicks.

Referring back to Olson's (2012) work and her case study of the Anonymous subgroup, Luzsec, she observed that the members of the group were privileged individuals but who conducted their operations as a small unit. When the results of the Lulzsec group's activities were disseminated though the wider Anonymous 
network, more support and abject pledges of allegiance to the original Lulzsec members soon followed. In effect the circles of influence within IRC that privilege extends to, varies hugely across time and the virtual spaces of IRC. While at some times (typically at the start of major operations) privileged nicks hold sway over large swaths of the nicks within the IRC, much of the time privilege within IRC is merely a demonstration of a persistent presence of an individual nick within an IRC channel.

This studies conclusion concerning privilege, re-orientates the model of social organization from the classic hierarchy to the more amorphous, self-ordering networks termed Netwar by Arquilla et al (Arquilla and Ronfeldt, 2000). This study has found that although Netwar was developed for non-state actor groups active within the physical world the model, with its leaderless, self-organizing networks is just as applicable to Anonymous. The main differentiator between a physical space Netwar group and a cyber space based Netwar group is the speed that the network changes, with cyber groups showing a far increased development cycle over groups occupying a purely physical space (the groups profiled in Kenny, 2008, showed maturation time scales of years being applies to groups active purely in physical space).

Perhaps it is hardly surprising that the ICT revolution, with its over focus on rapid communication, should facilitate groups like Anonymous and create its distinctive features of membership mobility (Rid, 2013) and leaderless organizational structure bound together by the so called 'strength of week ties' (Granovetter, 1973).

\section{About the author}

Stewart K. Bertram is an intelligence and security professional with a background in counter terrorism and military intelligence. Stewart specializes in analyzing the human factors associated with cyber security. Holding a Master of Letter in Terrorism Studies from St Andrews University and a Master of Science in Computing, Stewart combines both a social science and a hard science approach to cyber security.

\section{Annex A: Bibliography}

Kozinets, R. V. (2009). Netnography: Doing Ethnographic Research Online. 2009. London: SAGE Publications Ltd.

Time Staff. (2012). The 2012 Time 100 Poll. Time Magazine. Retrieved from http://www.time.com/time/ specials/packages/article/0,28804,2107952 2107953 2109562,00.html

Chomsky, N. (2012). Occupy. London: Penguin.

Bernays, E. (1928). Propaganda. Brooklyn: New York

Leyden, J. (2012). UK cops: How we sniffed out convicted AnonOps admin 'Nerdo.' The Register. Retrieved from http://www.theregister.co.uk/2012/12/14/uk anon investigation/ Date accessed 12 April 2012

Halupka, M (2011). The Evolution of Anonymous as a Political Actor. Retrieved from http://www.academia. edu/1220969/The Evolution of Anonymous as a Political Actor

Holden, J (2013). Deconstructing the Al-Qassam Cyber Fighters Assault on US Banks. Analysis Intelligence. Retrieved from http://analysisintelligence.com/cyber-defense/deconstructing-the-al-qassam-cyber-fightersassault-on-us-banks/

Olson, P. (2012). We Are Anonymous. Inside the Hacker World of Lulzsec, Anonymous, and the Global Cyber Insurgency. London: Little, Brown and Company 


\section{Journal of Terrorism Research}

Kumar, M. (2012). Anonymous Hacker take down GoDaddy with IRC Bots. The Hacker News. Retrieved from http://thehackernews.com/2012/09/anonymous-hacker-take-down-godaddy-with.html.

Parish, K. (2011). Suspected LulzSec Ringleader Arrested in Essex. Toms Hardware. 21 June 2011. Retrieved from http://www.tomshardware.com/news/LulzSec-Anonymous-hacktivist-Wickford-Essex-Ryan-

Cleary, 12982.html.

Cockerton, P.(2013). Anomymous hackers jailed for cyber attacks on financial firms and anti-piracy websites. The Mirror. Retrieved from http://www.mirror.co.uk/news/uk-news/anonymous-hackers-christopher-weatherhead-aka-1554188

Granovetter, M.(1973). TheStrength of Weak Ties. American Journal of Sociology 78(6) 1360-1380

Ghonim, W.(2012). Revolution 2.0. London: FourthEstate

Rid, T.(2013). Cyber War Will Not Take Place. London: Hurst

Holt, T.J., Strumsky,D., Smirnova, O., Kilger, M. (2012). Examining the Social Networks of Malware Writers and Hackers. International Journal of Cyber Criminology 6(1)

Décary-Hétu, D., Morselli, C. (2011). Gang Presence in Social Network Sites. International Journal of Cyber Criminology 5(1)

Pasmore, W. (1988). Designing Effective Organization. The Sociotechnical Systems Perspective. London: John Wiley \& Sons

Coleman, G. (2011). Anonymous: From the Lulz to Collective Action. NYU. Retrieved from http:// mediacommons.futureofthebook.org/tne/pieces/anonymous-lulz-Collective-action

Castells, M. (2001). The Internet Galaxy: Reflections on the Internet, Business, and Society. Cambridge: Oxford University Press.

Stryker, C. (2011). Epic Win for Anonymous: How 4Chan's Army Conquered the Web. London: Overlook Hardcover

Harriswow (2012). HOW TO JOIN ANONYMOUS-A BEGINNER'S GUIDE. Retrieved from http://www. youtube.com/watch?v=VPKRYs4nfsI

Nahai, N. (2012). Webs of Influence: The Psychology of Online Persuasion. Harlow: Pearson

Castelles, M. (2009). Communication Power. Oxford: Oxford University Press

Rheingold, H. (1993). The Virtual Community: Homesteading on the Electronic Frontier. Reading, MA: Addison-Wesley,

Itoh, H. (1992). Cooperation in Hierarchical Organizations: An Incentive Perspective. JLEO, V8 N2 321 anonbase (2011). Project Chanology. Retrieved from http://anonbase.wordpress.com/2011/03/19/projectchanology/

Correll, S. (2010). Operation:Payback broadens to “Operation Avenge Assange.” Retrieved from http:// pandalabs.pandasecurity.com/operationpayback-broadens-to-operation-avenge-assange/

J.D. McCarthy, M.N. Zald. (1973). The trend of social movements in America: professionalization and resource mobilization. Retrieved from http://deepblue.lib.umich.edu/handle/2027.42/50939

Robertson, A. (2013). LulzSec hacker Jeremy Hammond pleads guilty to role in Stratfor leak. Retrieved from http://www.theverge.com/2013/5/28/4372680/lulzsec-jeremy-hammond-pleads-guilty-to-role-in-stratforhack 


\section{Journal of Terrorism Research}

Pearce, J. (1980). Apathy or self interest? The volunteers' avoidance of leadership roles. Journal or Voluntary action Research, 9 , 85-90

Brown, H, M. (1989). Organizing Activity in the Woman's Movements. In B Klandermans (ed.), Organizing for Change. Greenwhich, CT: JAI Press

Lichterman, P. (1995). The Search for Political Community: American Activist Reinventing Commitment. Cambridge/ New York: Cambridge University Press

Melucci, A. (1996). Channeling Codes. Cambridge/ New York: Cambridge University Press

Diani, M. (2003). Leaders or Brokers? in Mario Diani and Doug McAdam (eds.), Social Movements and Networks. Oxford/ New York: Oxford University Press, 105-22

\section{Annex B: Generic hacker terms used throughout the paper}

- Nick - short for nickname, a nick is the title that a user posts comments under within the IRC server. Interchangeable with "Handle" a nick is common terminology within cyber ethnographic literature

- User - behind each nick is a user i.e. a physical person typing on a keyboard. The term User is separated from the term Nick due to the fact that multiple nicks can be associated with a single user and within the context of this research it was not possible to fully map each Nick to a User

- Channel Admins - user that have overall power on a channel, who typically act as arbiters in disputes, set social norms and have the power to bar nicks and even shut the whole channel down if they desire

- Lurkers - nicks that sit in a channel but do not post

- Privilege - assigned to nicks by a Channel Admin a privilege is a graphical symbol that is attached to a user's nick. A privileged nick has no more power to effect the flow of conversation within a channel than any other users, a privilege is in effect an honorific badge that designates that fact the nick carries influence within the channel

- Anon - identifies a member of Anonymous i.e. "I am an Anon." Used by many within the Collective as a method of self-identification

- Anonymous Collective-Anonymous and Anonymous Collective are used interchangeably. Many individuals involved in Anonymous would most likely reject the term Anonymous Collective however; the term has been used in many mainstream media article and has become standard phraseology when referring to Anonymous.

- Anonymous IRC and, IRC are used interchangeable within this paper. The author acknowledges that there are many more IRCs than the ones associated with Anonymous however, within the scope of this paper the reader should infer that IRC refers to Anonymous IRC. (all definitions taken from Bertram, 2013) 


\section{Journal of Terrorism Research}

